\title{
Clinical usefulness of urine cytology in the detection of bladder tumors in patients with neurogenic lower urinary tract dysfunction
}

This article was published in the following Dove Press journal: Research and Reports in Urology

\section{Jürgen Pannek Franziska Rademacher Jens Wöllner}

Neuro-Urology, Swiss Paraplegic Center, Nottwil, Switzerland
Correspondence: Jürgen Pannek Chefarzt Neuro-Urologie, Schweizer Paraplegiker-Zentrum, Guido A. Zäch Strasse I, CH - 6207 Nottwil, Switzerland

Tel +4 I 4I 9395924

Fax +4| 4| 9395923

Email juergen.pannek@paraplegie.ch
Introduction: Screening for bladder cancer in patients with neurogenic lower urinary tract dysfunction is a challenge. Cystoscopy alone is not sufficient to detect bladder tumors in this patient group. We investigated the usefulness of combined cystoscopy and urine cytology.

Materials and methods: By a systematic chart review, we identified all patients with neurogenic lower urinary tract dysfunction who underwent combined cystoscopy and urine cytology testing. In patients with suspicious findings either in cytology or cystoscopy, transurethral resection was performed.

Results: Seventy-nine patients (age 54.8 \pm 14.3 years, 38 female, 41 male) were identified; 44 of these used indwelling catheters. Cystoscopy was suspicious in 25 patients and cytology was suspicious in 17 patients. Histologically, no tumor was found in 15 patients and bladder cancer was found in 6 patients. Sensitivity for both cytology and cystoscopy was $83.3 \%$; specificity was $43.7 \%$ for cytology and $31.2 \%$ for cystoscopy. One bladder tumor was missed by cytology and three tumors were missed by cystoscopy. If a biopsy was taken only if both findings were suspicious, four patients would have been spared the procedure, and one tumor would not have been diagnosed.

Conclusion: A combination of cystoscopy and urine cytology can improve bladder tumor detection rates and lower the number of unnecessary biopsies.

Keywords: bladder cancer, neurogenic lower urinary tract dysfunction, urine cytology, cystoscopy

\section{Introduction}

Bladder cancer is a relatively rare malignant disease in both the general population and in persons with spinal cord injury (SCI). ${ }^{1}$ However, the bladder cancer mortality ratio is about 6.7 times higher in persons with SCI. ${ }^{2}$

Although there is consensus that bladder cancer in persons with SCI is associated with significant mortality, there is no concise surveillance strategy. ${ }^{3}$ In a recent meta-analysis, the sensitivity of surveillance cystoscopy and cytology for detecting bladder cancer was $64 \%$ and $36.3 \%$, respectively. ${ }^{3}$ Especially in patients with longterm indwelling catheters, screening is a challenge. On one hand, patients with an indwelling catheter usage for at least 16 years have the highest risk for bladder cancer. ${ }^{3}$ On the other hand, especially long-term indwelling catheters may cause morphologic alterations that are impossible to distinguish from bladder cancer by cystoscopy alone. To perform transurethral biopsy in each patient creates a significant burden for the patient, is not without complications, and is expensive. 
Therefore, we aimed to assess the clinical usefulness of a combination of cystoscopy and urine cytology for bladder cancer screening in patients with SCI.

\section{Materials and methods}

By a systematic chart review of all patient charts beginning in 2014 (implementation of urine cytology at our hospital), we identified all patients with a neurogenic lower urinary tract dysfunction (NLUTD) who underwent combined cystoscopy and urine cytology testing between 2014 and 2017 at our institution. In our department, every patient with an indwelling catheter for at least 5 years, or any patient with NLUTD and hematuria or with suspicious finding in ultrasound of the bladder underwent this procedure. Retrospective data collection was approved by the local ethics committee (Ethikkommission Nordwest- und Zentralschweiz). Due to the retrospective nature of the analysis, informed patient consent was not requested. The patient data were collected and stored in a database made anonymous, thus ensuring data confidentiality.

Cystoscopy was performed yearly as video-endoscopy with a flexible cystoscope by one of four experienced, board-certified urologists. Prior to cystoscopy, urine was sampled by bladder wash and cytology was performed by a single pathologist with a substantial clinical experience in urine cytology and bladder histology. In brief, the bladder was rinsed before cystoscopy to collect the urine for cytology. A thiomersal-containing solution (Cytorich ${ }^{\mathrm{TM}}$; Thermo Fisher Scientific, Waltham, MA, USA) was added for urine asservation. Papanicolaou stain was used, and three slides from each case were examined (Figure 1). For evaluation, a light microscope in various magnifications $(40 \times, 100 \times$, $200 \times, 400 \times$ ) was used.

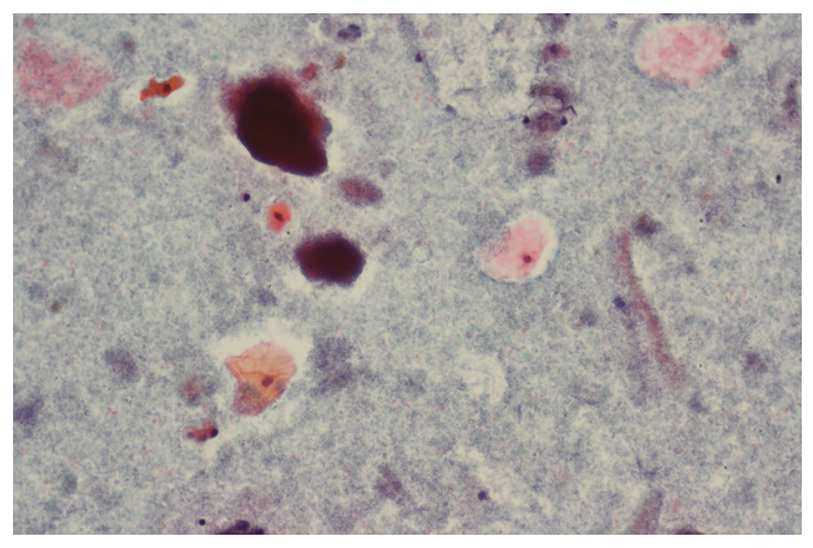

Figure I Urothelial cells suspicious for urothelial carcinoma, normal urothelial cells, and bacterial colonization (Papanicolaou stain, 200x).
In patients with no suspicious cells in the urine cytology and inconspicuous cystoscopy, no further diagnostic procedures were performed. In patients with suspicious findings either by cytology or cystoscopy, transurethral resection of the finding was performed in the operating room.

Statistical data analysis (sensitivity and specificity) was performed using IBM SPSS Statistics Version 24 (2016).

\section{Results}

Between October 2014 and January, 2017, 79 patients (age $54.8 \pm 14.3$ years, 38 female, 41 male) who underwent combined cystoscopy and urine cytology were identified. NLUTD was caused by spinal cord lesions in 65 patients, multiple sclerosis in 8 patients, and by other neurologic disorders in 6 patients. Of the patients with SCI, 23 suffered from a cervical lesion, 37 from a thoracic lesion, and 5 from a lumbar lesion. The demographic data and the levels of injury are summarized in Table 1.

Indications for the mentioned work-up were hematuria, suspicious findings in ultrasound of the bladder, or the presence of an indwelling catheter for 5 years or longer, as screening after this time span is common clinical practice. ${ }^{4}$

\section{Bladder management}

An indwelling catheter (transurethral: $\mathrm{n}=18$; suprapubic: $\mathrm{n}=26$ ) was used in 44 patients $(55.7 \%)$ for a median duration of $7.4 \pm 7.3$ years. Eighteen patients (22.8\%) used intermittent catheterization and 17 patients (21.5\%) applied reflex voiding.

\section{Cystoscopy}

In 25 patients, cystoscopy revealed suspicious findings. In eight of these patients, the urologist was sure by morphologic criteria that the patient suffered from bladder cancer. In four of these eight patients, both cytology and histology confirmed an urothelial tumor and in three cases, both cytology and biopsy were negative. In one case, a bladder cancer (pTa G1) was detected, but cytology was negative.

Table I Demographic results

\begin{tabular}{llll}
\hline Demographic & Patients (n) & Mean \pm SD & Range \\
\hline Male gender & $4 \mathrm{I}$ & & \\
Female gender & 38 & & \\
Age (years) & 79 & $54.8 \pm 14.3$ & $25-88$ \\
NLUTD duration (years) & 79 & $16.3 \pm 13.4$ & $\mathrm{I}-55$ \\
Indwelling catheter (years) & 44 & $7.4 \pm 7.3$ & $\mathrm{I}-28.5$ \\
Complete SCl & 35 & & \\
Incomplete SCl & 30 & & \\
Other etiology & $\mathrm{I}$ & & \\
\hline
\end{tabular}

Abbreviations: NLUTD, neurogenic lower urinary tract dysfunction; SCl, spinal cord injury; SD, standard deviation. 


\section{Cytology}

In 17 patients, suspicious/malignant cells were detected in urine cytology.

\section{Histologic results}

In all 25 patients with suspicious cystoscopic findings, transurethral resection of the bladder was recommended. Due to the broad-based bullous findings in all patients, cold-cup biopsies were not sufficient to safely exclude malignant growth. Three patients refused to undergo this intervention. Therefore, transurethral resection of the bladder (either resection of a visible structure or quadrant resection biopsy) was performed in 22 patients within 6 weeks after the initial cystoscopy. At histologic examination of the 22 specimens retrieved, no tumor was found in 15 patients, whereas bladder cancer (low grade [n=2] or high-grade $[\mathrm{n}=3]$ urothelial cancer, muscle-invasive squamous cell carcinoma $[\mathrm{n}=1]$ ) was found in 6 patients (Table 2 ).

\section{Usefulness of diagnostic procedures}

Sensitivity of cytology and cystoscopy compared to biopsy was $83.3 \%$ for both procedures; specificity was $43.7 \%$ for cytology and $31.25 \%$ for cystoscopy. Only one patient with a non-muscle-invasive bladder tumor had a normal cytology, and would therefore have been missed if we had used cytology alone. If we relied exclusively on cystoscopy, three bladder tumors would have been missed. If a biopsy was taken only when both cytologic and cystoscopic findings were suspicious, four patients would have been spared the procedure and one tumor would not have been diagnosed (Table 3).

\section{Risk factors for bladder cancer}

Three of the six patients with bladder cancer used a suprapubic catheter, whereas two used intermittent catheterization and one performed reflex voiding. Time between onset of SCI and diagnosis of bladder cancer was 23.7 years in all patients with tumors and 5.6 years in the three patients with suprapubic catheters.

\section{Discussion}

Screening for bladder tumors in patients with NLUTD remains a challenge, and no generally accepted strategy

Table 2 Histologic results ( $n=22$, patients with bladder biopsy)

\begin{tabular}{lll}
\hline Histologic result & Patients (n) & Patients (\%) \\
\hline No tumor & 16 & 72.7 \\
Low-grade urothelial cancer & 2 & 9.1 \\
High-grade urothelial cancer & 3 & 13.6 \\
>pT3 squamous cell carcinoma & $\mathrm{I}$ & 4.5 \\
\hline
\end{tabular}

Table 3 Diagnostic tables

\begin{tabular}{lllll}
\hline & Findings & \multicolumn{2}{l}{ Histologic findings } & Total \\
\cline { 2 - 4 } & & + & - & \\
\hline Cystoscopy & + & 5 & 11 & 16 \\
& - & 1 & 5 & 6 \\
& Total & 6 & 16 & 22 \\
\hline Washing cytology & + & 5 & 9 & 14 \\
& - & 1 & 7 & 8 \\
& Total & 6 & 16 & 22 \\
\hline
\end{tabular}

Note: +, positive finding (tumor); -, negative finding (no tumor).

exists. ${ }^{3}$ According to the results of our study, urine cytology can aid in the detection of bladder tumors and in decreasing the number of unnecessary biopsies.

Due to the low incidence of bladder tumors, the rationale for a general screening in persons with SCI is under debate. Some authors argue that the risk of dying from bladder cancer is not high enough to warrant screening, even in subpopulations with possible risk factors, for example, with indwelling catheters. Instead, they recommend to identify risk factors that warrant screening. ${ }^{5}$ In contrast, others advocate that SCI in itself is a risk factor for bladder cancer development and, therefore, recommend screening cystoscopy in every patient with NLUTD due to SCI. ${ }^{6,7}$ Without surveillance, we would have missed six of eight bladder cancers, three of them being muscle invasive and, as a consequence, potentially fatal. Therefore, we propose to perform screening at least in patients with established risk factors.

Defining risk factors for bladder cancer in patients with SCI, however, is not an easy task. In a recent meta-analysis, it was demonstrated that in comparison to the general population, patients with SCI and bladder cancer are younger (50 vs 73 years), and squamous cell carcinoma seems to be more frequent $(36.8 \% \mathrm{vs} 7 \%)$ in this group of patients. ${ }^{3}$ The mean interval between SCI and bladder cancer development was 24 years. Long-term indwelling catheters seem to be a risk factor. This is highlighted by the comparatively high cancer detection rate (6/75 patients, $8 \%)$ in our group, which consisted mainly of patients with long-term indwelling catheters. Generally, having an indwelling catheter for more than 10 years is considered to increase the risk for developing bladder cancer; ${ }^{4,8,9}$ but in the mentioned meta-analysis, a time span of even 16 years was found. ${ }^{3}$ This is corrupted by the fact that the data on which this calculation is based all derive from retrospective studies, thus the exact time span cannot reliably be determined. In our study, the time between onset of SCI and diagnosis of bladder cancer was 5.6 years in the 
group of patients with a suprapubic catheter and 23.7 years in those without an indwelling catheter, but our group of patients was too small to draw any firm conclusions from this fact.

Besides indwelling catheters (transurethral or suprapubic), the NLUTD itself, frequently causing chronic bacteriuria and bladder stones, immune system changes, years since injury, ${ }^{10}$ and injury severity ${ }^{2}$ are regarded as possible risk factors for bladder cancer, which are different from those in the general population. In addition, risk factors not directly related to SCI, such as smoking, are also present in patients with SCI. ${ }^{10}$ We did not ask for smoking habits, as cancer detection, rather than pathophysiology, was the main topic of the study. In summary, due to the scarce existing robust data, we propose that at least patients with long-term indwelling catheters should be screened and patients with gross hematuria should undergo a meticulous work-up.

The best way to perform bladder cancer screening is still under debate. Hematuria, detected by urinalysis, can be caused by various reasons, especially in persons with indwelling catheters. Therefore, the interpretation is challenging and urinalysis alone is not recommended as a screening tool. ${ }^{6}$ Bladder ultrasound has been demonstrated to be inferior to cystoscopy in the diagnosis of bladder tumors. ${ }^{11}$

Several studies demonstrated that screening cystoscopy alone is not sufficiently sensitive and specific to be used as a surveillance tool in SCI patients. Yang and Clowers did not detect a single tumor in 59 patients undergoing a total of 156 cystoscopies, the majority of which had to be performed under general anesthesia, ${ }^{12}$ as especially patients with an SCI above the level of Th10 are at risk for autonomic dysreflexia, a potentially life-threatening complication. ${ }^{13}$ Particularly in patients with indwelling catheters, bladder tumor detection merely based on the endoscopic appearance is virtually impossible, as it is not possible to reliably distinguish the morphologic alterations caused by the catheters from bladder tumors. Moreover, a cystoscopic evaluation in every patient will cause additional costs. As a consequence, we do not support the idea of sequential cystoscopies in each patient with NLUTD.

Although the use of cytology to diagnose bladder cancer can be limited by several factors, including, but not limited to, inflammation, degenerative changes, instrumentation effect, or lithiasis, ${ }^{14}$ in our series, urine cytology provided the aforementioned promising results, which may at least in part be due to the fact that is was performed by a single experienced uropathologist. ${ }^{15}$ Cytology is especially sensitive for detecting high-grade tumors, which are potentially fatal.
Therefore, its use is recommended for high-risk tumors. ${ }^{16}$ In our study, we found it helpful to distinguish reactive urothelial alterations from low-grade tumors as well.

The usefulness of our approach, that is, combining cystoscopy and cytology, is supported by other authors. In a recent meta-analysis, cystoscopy demonstrated a sensitivity of $64 \%$ for detecting bladder cancer, whereas cytology demonstrated a sensitivity of $36.3 \% .{ }^{3}$ Sammer et al detected 13 suspicious findings in 129 patients with NLUTD, predominantly due to SCI, using this combination. In these 13 patients, biopsies were performed, diagnosing a bladder tumor in one patient. However, they did not describe whether biopsies were performed due to cystoscopic findings or due to cytology. ${ }^{6}$ The authors concluded that screening cystoscopy and cytology should be performed in every patient with NLUTD after 5 years every 1-2 years. Stonehill et al evaluated 906 cytology specimens in a cohort of 208 patients with SCI. Seventeen bladder cancers were detected, leading to a specificity of $71 \%$. However, they performed repetitive (as a rule yearly) cytologies; thus, in patients with bladder cancer, the diagnosis may be delayed if only patients with repetitively positive cytologies were further evaluated. ${ }^{17}$

In a study comparing cystoscopy to different bladder cancer detection tests, including urine cytology, none of the mentioned tests improved tumor detection. ${ }^{18}$ The authors concluded that cystoscopy remains the gold standard for bladder cancer surveillance in SCI patients. However, bladder tumor detection rate in their study was low; the analysis was based on 3 bladder cancer patients detected in a cohort of 457 persons with SCI. In a pilot study, no correlation of urinary BLCA-4 with a history of urinary tract infection, smoking, catheterization, or cystitis was demonstrated; therefore, the BLCA-4 assay may be useful for bladder cancer screening in patients with SCI in the future. ${ }^{19}$

In patients who seem to belong to a risk group, that is, patients with indwelling catheters, random bladder biopsies were proposed. ${ }^{4}$ The recommendation, however, was based on a study including only 36 patients. As this procedure requires general anesthesia, at least in tetraplegic patients and in patients with an incomplete SCI, benefits and possible harms should be evaluated in each individual case. Authors claim usefulness of bladder biopsies in patients with a high risk of bladder cancer, for example, with recurrent gross hematuria. ${ }^{20}$ However, invasiveness and cost of this approach prevent its use as a screening procedure, and bladder biopsy is only recommended if cystoscopy and/or cytology revealed suspicious findings. 


\section{Conclusion}

Whereas a general screening for bladder cancer in patients with SCI does not seem to be justified, surveillance in certain risk groups may be feasible. Patients with indwelling catheters may be such a group at risk. The heterogeneity of the different studies summarized in the meta-analyses available makes it difficult to develop prevention, screening, and management strategies. Therefore, despite the existence of meta-analyses, studies focusing on certain subgroups, as for example, our study, are important to evaluate the usefulness of screening tools under certain conditions. According to our data, a combination of cystoscopy and urine cytology is minimally invasive, and the combination of the two techniques can improve detection rates and lower the number of unnecessary biopsies.

\section{Disclosure}

The authors report no conflicts of interest in this work.

\section{References}

1. Torre LA, Bray F, Siegel RL, Ferlay J, Lortet-Tieulent J, Jemal A. Global cancer statistics, 2012. CA Cancer J Clin. 2015;65(2):87-108.

2. Nahm LS, Chen Y, DeVivo MJ, Lloyd LK. Bladder cancer mortality after spinal cord injury over 4 decades. JUrol. 2015;193(6):1923-1928.

3. Gui-Zhong L, Li-Bo M. Bladder cancer in individuals with spinal cord injuries: a meta-analysis. Spinal Cord. 2017;55(4):341-345.

4. Hamid R, Bycroft J, Arya M, Shah PJ. Screening cystoscopy and biopsy in patients with neuropathic bladder and chronic suprapubic indwelling catheters: is it valid? J Urol. 2003;170(2 Pt 1):425-427.

5. Elliott SP. Screening for bladder cancer in individuals with spinal cord injury. J Urol. 2015;193(6):1880-1881.

6. Sammer U, Walter M, Knüpfer SCMehnert U, Bode-Lesniewska B, Kessler TM. Do we need surveillance urethro-cystoscopy in patients with neurogenic lower urinary tract dysfunction? PLoS One. 2015;10(10):e0140970.
7. Kalisvaart JF, Katsumi HK, Ronningen LD, Hovey RM. Bladder cancer in spinal cord injury patients. Spinal Cord. 2010;48(3):257-261.

8. Pannek J. Transitional cell carcinoma in patients with spinal cord injury: a high risk malignancy? Urology. 2002;59(2):240-244.

9. Stonehill WH, Dmochowski RR, Patterson AL, Cox CE. Risk factors for bladder tumors in spinal cord injury patients. $J$ Urol. 1996;155(4): $1248-1250$

10. Welk B, McIntyre A, Teasell R, Potter P, Loh E. Bladder cancer in individuals with spinal cord injuries. Spinal Cord. 2013;51(7):516-521.

11. Stamatiou K, Papadoliopoulos I, Dahanis SZafiropoulos G, Polizois $\mathrm{K}$. The accuracy of ultrasonography in the diagnosis of superficial bladder tumors in patients presenting with hematuria. Ann Saudi Med. 2009;29(2):134-137.

12. Yang CC, Clowers DE. Screening cystoscopy in chronically catheterized spinal cord injury patients. Spinal Cord. 1999;37(3):204-207.

13. Liu N, Fougere R, Zhou MW, Nigro MK, Krassioukov AV. Autonomic dysreflexia severity during urodynamics and cystoscopy in individuals with spinal cord injury. Spinal Cord. 2013;51(11):863-867.

14. Caraway NP, Katz RL. A review on the current state of urine cytology emphasizing the role of fluorescence in situ hybridization as an adjunct to diagnosis. Cancer Cytopathol. 2010;118(4):175-183.

15. Janzen J, Bersch U, Pietsch-Breitfeld BPressler H, Michel D, Bültmann B. Urinary bladder biopsies in spinal cord injured patients. Spinal Cord. 2001;39(11):568-570.

16. Yafi FA, Brimo F, Steinberg J, Aprikian AG, Tanguay S, Kassouf W Prospective analysis of sensitivity and specificity of urinary cytology and other urinary biomarkers for bladder cancer. Urol Oncol. 2015;33(2):e25-e31.

17. Stonehill WH, Goldman HB, Dmochowski RR. The use of urine cytology for diagnosing bladder cancer in spinal cord injured patients. J Urol. 1997;157(6):2112-2114.

18. Davies B, Chen JJ, McMurry T, et al. Efficacy of BTA stat, cytology, and survivin in bladder cancer surveillance over 5 years in patients with spinal cord injury. Urology. 2005;66(4):908-911.

19. Konety BR, Nguyen TS, Brenes G, et al. Clinical usefulness of the novel marker BLCA-4 for the detection of bladder cancer. $J$ Urol. 2000;164(3 Pt 1):634-639.

20. Vaidyanathan S, Soni BM, Singh G, Hughes PL, Mansour P, Oo T. Delay in diagnosis of cancer as a patient safety issue - a root cause analysis based on a representative case report. Patient Saf Surg. 2011;5:19.
Research and Reports in Urology

\section{Publish your work in this journal}

Research and Reports in Urology is an international, peer-reviewed, open access journal publishing original research, reports, editorials, reviews and commentaries on all aspects of adult and pediatric urology in the clinic and laboratory including the following topics: Pathology, pathophysiology of urological disease; Investigation and treatment of

\section{Dovepress}

urological disease; Pharmacology of drugs used for the treatment of urological disease. The manuscript management system is completely online and includes a very quick and fair peer-review system, which is all easy to use. Visit http://www.dovepress.com/testimonials.php to read real quotes from published authors. 